La cybersanté oui, mais comment? On nous avait promis que l'informatique nous simplifierait la vie, mais on a assisté à une tentative de maîtrise de l'administration et du pouvoir politique sur tout, avec une inflation invraisemblable de la paperasserie. Alors à nous d'orienter la suite!

Dr Monique Gauthey,

membre du Comité central, responsable du domaine eHealth

\title{
La cybersanté au service des patients et des médecins
}

En fait, nous savons bien ce qu'il nous faudrait: un accès rapide et aisé aux données importantes et nécessaires pour les traitements, une alternative pratique aux recherches sans fin et aux coups de fil visant à localiser tel ou tel résultat d'examen, des formulaires préremplis facilitant nos tâches ... Mais tout cela semble bien loin pour l'instant.

Le groupe de travail eHealth de la FMH (GT) s'est fixé la tâche ambitieuse de faire en sorte que les nombreuses activités en cours dans le domaine e-health soient ciblées sur les besoins des patients et des médecins. La première démarche du GT a consisté à élaborer des propositions pour une stratégie e-health de la FMH pour la période 2008-2012, basées sur les objectifs stratégiques de la FMH. De nature générale, ces propositions sont orientées sur le moyen terme. Dans une deuxième étape, le GT formule une prise de position sur divers thèmes d'actualité de la cybersanté tels que les dossiers électroniques de patient, cartes d'assuré ou de santé, etc.

L'objectif central de cette stratégie de la FMH est de créer un environnement e-health ayant une utilité pratique pour le patient et le médecin. Pour influencer activement les développements dans ce secteur, il nous faut être représentés dans les commissions importantes, mais aussi connaître les besoins du corps médical et des patients afin de les faire valoir vis-à-vis de l'extérieur. En partant des objectifs et besoins ainsi identifiés, nous pouvons et devons ensuite élaborer en continu nos positions sur les divers thèmes actuels.

La mise en réseau des membres de la FMH est également importante: il ne faut pas que chacune et chacun doive faire seul l'expérience, parfois pénible, d'une introduction ou d'une adaptation. Souvent ce n'est pas l'outil qui est décisif, mais la façon dont on s'en sert. Les mesures à prendre pour assurer la réutilisation et la valorisation ultérieure des données peuvent s'avérer plus ou moins simples.

Les objectifs suivants sous-tendent l'élaboration de la stratégie e-health de la FMH:

\section{Renforcer la sécurité des patients}

L'e-health doit accorder la priorité à la sécurité des patients et aux mesures appropriées pour la garantir en tout temps, p. ex. pour assurer la sauvegarde des données ou éviter de fausses saisies. A l'inverse, il est possible de tirer parti de certains outils électroniques de façon ciblée comme par exemple la prescription médicamenteuse électronique - afin de garantir la qualité.

\section{Mettre le traitement au centre}

Le traitement du patient doit rester au centre des préoccupations et être soutenu. Les outils de l'e-health devraient donc surtout servir à faciliter l'échange d'information tout au long de la chaîne de traitement.

\section{Garantir la protection des données,} la sphère privée et l'autonomie

Une protection des données efficace et préservant la sphère privée constitue pour nous le préalable indispensable à l'acceptation de l'e-health. De même, la libre gestion de la relation médecin-patient devra rester garantie dans le cadre de l'e-health, malgré les modifications que cette relation connaîtra certainement.

\section{Proposer de la formation}

Il nous faut veiller à une intégration appropriée de l'e-health et de l'informatique médicale dans la formation des médecins. Ceux-ci ne devront pas devenir des spécialistes de l'informatique, mais ils devraient acquérir les connaissances et aptitudes leur permettant d'utiliser correctement les outils disponibles. Et les patients devraient en savoir suffisamment en matière d'e-health pour pouvoir décider eux-mêmes ce qui advient de leurs données. Une transmission qualitativement adéquate du savoir médical peut aussi être encouragée.

\section{Financement}

Last but not least, la question du financement - en particulier celui de l'infrastructure du cabinet médical - est absolument cruciale. Pour «démarrer le convoi» et atteindre une masse critique dans les cabinets, un financement incitatif initial sera indispensable en plus d'une tarification appropriée.

Si nous atteignons ces objectifs, nous aurons fait un grand pas vers une cybersanté utile au traitement des patients.

Judith Wagner, responsable du département eHealth de la FMH 